\title{
No coordination required for resources allocation during colony fission in a social insect? An individual-based model reproduces empirical patterns
}

François Lavallée ${ }^{1,2 *}$, Guillaume Chérel ${ }^{2 *}$, Thibaud Monnin ${ }^{3}$

1 Université Clermont Auvergne, INRAe, UR LISC, Centre de Clermont-Ferrand, 63178 Aubière, France

2 Complex Systems Institute of Paris île-de-France, 113 rue Nationale, 75013, Paris, France

3 Sorbonne Université, Université Paris Est Créteil, Université de Paris, CNRS, INRAe, IRD, Institute of Ecology and Environmental sciences of Paris, iEES Paris, 75005 Paris, France

* these authors contributed equally

Corresponding author: thibaud.monnin@sorbonne-universite.fr

ORCID numbers: GC: 0000-0002-5289-649X; TM: 0000-0003-3762-942X

\section{ABSTRACT:}

Social insects are classic examples of cooperation and coordination. For instance, laboratory studies of colony relocation, or house-hunting, have investigated how workers coordinate their efforts to swiftly move the colony to the best nesting site available while preserving colony integrity, i.e. avoiding a split. However, several studies have shown that, in some other contexts, individuals may use private rather than social information and may act solitarily rather than in a coordinated way. Here, we study resource allocation by a mature ant colony when it reproduces by fissioning into several colonies. This is a very different task than house-hunting in that colony fission seeks the split of the colony. We develop a simple individual-based model to test if colony fission and resource allocation may be carried out by workers acting solitarily with no coordination. Our model reproduces well the pattern of allocation observed in nature (number and size of new colonies). This does not show that workers do not communicate nor coordinate. Rather, it suggests that independent decision making may be an important component of the process of resource allocation.

KEYWORDS: decision making, ants, Cataglyphis piliscapa, NetLogo, model calibration, OpenMole

\section{DECLARATIONS:}

- Funding: Not applicable

- Conflicts of interest/Competing interests: Not applicable

- Ethics approval: Not applicable

- Consent to participate: Not applicable

- Consent for publication: Not applicable

- Availability of data and material: Not applicable

- Code availability: provided as supplementary material

- Authors' contributions: TM wrote the NetLogo model, FL and GC carried out the simulation analysis, all authors jointly wrote the article

ACKNOWLEDGMENTS: We thank two anonymous referees for their valuable comments on an earlier version of the manuscript. 


\section{INTRODUCTION}

Insect colonies are highly integrated entities capable of accomplishing complex tasks, such as building large nests and choosing the best food source or nesting site (Franks et al. 2002; Visscher 2007; Sasaki and Pratt 2018). This capacity results from the cooperative behaviour of many individual workers. How individual behaviours based on partial information scale up to produce an effective colony-level behaviour has been the focus of many studies. House-hunting, that is, choosing a new nesting site where to relocate the colony, has proven to be a very fruitful bioassay to investigate this (Franks et al. 2002; Visscher 2007). In laboratory colonies of Temnothorax ants, emergency colony relocation may be triggered by removing the roof of the nest. Colony relocation is then organised by a group of experienced scout workers, who seek a potential nesting site in the surroundings of the nest (Richardson et al. 2018). Each scout visits and evaluates the nesting sites she finds. She rejects inappropriate ones (i.e. below a threshold of quality) until finding one that is good enough, at which point she switches behaviour and starts leading other scouts to that nesting site by means of tandem runs (Robinson et al. 2009, 2011). When the scouts present at a nesting site reach a threshold number (a quorum), they again switch behaviour and start relocating the colony to that nesting site by carrying colony members in their mandibles (Pratt et al. 2002).

Here, we investigate how the behaviours of workers determine the colony-level behaviour in another context, namely during colony foundation by colony fission. Colony fission has evolved repeatedly in social insects and is widespread. It consists in a mature colony producing new colonies by means of splitting, i.e. when one or more queens depart the mature colony with workers to initiate new colonies (Cronin et al. 2013). In addition, the splitting (and fusioning) of nests is also central to the ecology of many social insect species whose colonies inhabit multiple nests simultaneously. For instance, in the Peak District (UK), large colonies of the wood ant Formica lugubris are spread among ten interconnected nests (range 4-20) whose number and size they adjust to best exploit the resources available in the environment (Burns et al. 2020, 2021). Here, we analyse how a mature colony allocates its resources when it divides itself into several new colonies. Unlike in house-hunting, where the relocating colony seeks to maintain its integrity by avoiding an accidental split, a fissioning colony's aim is to split into several new colonies, and how it partitions its resources among them is an important determinant of its fitness.

Complex mechanisms may govern resource allocation during colony fission. However, we study here the parsimonious alternative that it may be achieved, at least in part, by workers using private information and acting solitarily. This possibility is supported by several lines of evidence. Firstly, just because colony fusion looks well organized does not mean that it is socially coordinated. For instance, brood transport can be synchronized by environmental cues but carried out by workers acting solitarily (Mersch et al. 2018). Secondly, the use of private information is well documented in ants. For instance, the common black garden ant, Lasius niger, combines private and social information during foraging (Grüter et al. 2011; Czaczkes et al. 2015, 2016) and house hunting ants visiting potential nesting sites combine private and social information before choosing one (Cronin 2013; Stroeymeyt et al. 2017). Fissioning colonies may allocate their resources without workers visiting and monitoring the size of all newly founded colonies, that is, without having global information, as occurs in foraging greenhead ants, Rhytidoponera metallica, where foragers can select the best food source without comparing all available food sources (Dussutour and Nicolis 2013). Thirdly, although social information is of high value it may be more efficient to rely on private information under some circumstances, such as when it is of high quality (Stroeymeyt et al. 2017), or 
when social information is outdated (Dechaume-Moncharmont et al. 2005) or conflicting with private information (Cronin 2013).

We implement the simplest possible individual-based model of colony fission and resource allocation, where workers solely use private information and act in an uncoordinated way. The model is suited to species who do not use chemical trails, i.e. where foragers rely on private information. It comprises environmental, colony-level and individual-level parameters, four of which are studied here while others are held fixed. Each forager decides on her own at which potential nesting site to found a new colony, and she transports resources to that nesting site only. Therefore, foragers as a collective may select one or several nesting sites, thereby resulting in the foundation of one or several new colonies.

We then test whether this model can reproduce well the pattern of allocation observed in nature in the ant Cataglyphis piliscapa (previously C. cursor var. piliscapa), for which there are data on the number and size of colonies produced by fission (Lenoir et al. 1988; Chéron et al. 2011). Fissioning colonies vary markedly in size ( $731+$ +- 456 workers, mean +/- SD, ranging from 252 to 1,808 workers), in the number of workers they invest in the production of new colonies $(519+/-382$ workers, range 180 to 1,759), and in the number of new colonies produced (one to seven, four on average) (Chéron et al. 2011). Cataglyphis ants do not use chemical trails. Instead, they use path integration and visual landmarks for navigating outside their nest (Collett et al. 1998; Boulay et al. 2017). Owing to the lack of chemical trails, inexperienced workers cannot orientate outside the nest and hence cannot join a newly founded colony on their own. Resource allocation is therefore carried out by the foragers, that is, older workers that leave the nest to collect food and that have learned to orientate outside. Foragers transport the brood and inexperienced workers one at a time in their mandibles, from the mature colony to the new colonies, by means of numerous back-and-forth trips (Lenoir et al. 1988; Chéron et al. 2011). Using a parameter inference procedure and the empirical data available on C. piliscapa, we construct a model which reproduces the data for each of the 19 colonies in our data and determine the credible values of the four parameters studied. Success of the model in reproducing well the observed data would support the idea that workers acting solitarily without coordination may play an important role in colony fission. In addition, performing a parameter inference independently for each colony may shed light on possible differences in colony traits associated with different allocation patterns, for instance, between colonies producing few versus many new colonies.

\section{MATERIAL \& METHODS}

Individual-based model

We use a spatially explicit individual-based model implemented with NetLogo 6.2.0 (Wilensky and Evanston 1999). An overview of the model is provided below using the ODD protocol (Grimm et al. 2010). A sketched algorithm is in Fig. 1, and the NetLogo code is in supplementary material.

\section{Purpose}

We model how a social insect colony splits into one or more new colonies and allocates resources among them. Foragers are responsible for the choice of nesting sites and the allocation of resources, and they do it using private information only and without coordination with one another. 


\section{Entities, state variables, and scales}

The model comprises three types of agents: the initial colony, which has a given size that consists of a percentage of foragers and of resources (representing non-foraging workers and brood), with a set amount of resources to be allocated to new colonies; nests (i.e. available nesting sites), which are characterised by their quality and location; foragers, with each forager visiting some nests she then memorises.

\section{Process overview and scheduling}

The model goes through two separate phases. The exploring phase mimics the fact that, in the days or weeks preceding colony fission, foragers acquire knowledge of the environment while collecting food. This phase consists of foragers independently wandering randomly in the environment, assessing the quality of the nesting sites they encounter, and choosing one following one of three choice strategies (see Submodels below). Several foragers may independently choose the same nesting site. This exploration phase lasts a set duration.

The second phase is the allocation of the resources of the initial colony to new colonies. Foragers who found no nesting site during the preceding exploring phase become resources (i.e. they will be transported to a nest by an informed forager), and foragers who found a nesting site start the second phase at the initial colony. Each forager transports resources to the nesting site she has chosen. She transports resources one at a time, through numerous back-and-forth trips between the initial colony and the nesting site. When the initial colony is depleted of resources, each forager joins the nesting site she has selected and built up into a new colony. The simulation ends when all foragers have allocated themselves to a new colony, that is, when all foragers and allocated resources have been partitioned to found one or several new colonies. Note that the two phases do not have the same time scale. The exploration phase mimics weeks of information gathering, whereas the allocation phase lasts one or a few days in nature (Chéron et al. 2011).

\section{Design concepts}

- Basic principles: the model assumes that all foragers are identical, which is a common assumption (Dechaume-Moncharmont et al. 2005). It assumes that they follow the same pattern of random movement (same speed and sinuosity), are equally susceptible to committing errors when estimating nesting site quality, and make similar errors. In particular, it assumes that all foragers explore potential nesting sites. It may be that this task is carried out by a sub-group of scouts in some species, but we have no evidence for this in our model species $C$. piliscapa. In addition, the model assumes that foragers acquire knowledge of the environment days or weeks before undertaking resource allocation. This has been shown in Temnothorax, where foragers can assess and remember the quality of nesting sites they come across while foraging at a time when their colony is not about to relocate (Franks et al. 2007).

- Emergence: the number of new colonies produced and the amount of resources each receives emerge from the behaviour of foragers

- Objectives: each forager independently chooses the nesting site with the highest assessed quality among those she has visited. This assessment is made with an error that is proportional to quality. The assessed quality is drawn from a normal distribution of mean quality and standard deviation quality * nest_quality_assessment_error, where nest_quality_assessment_error is a percentage of quality. That is, the assessment error is larger for high quality nesting sites, which is realistic in that foragers may find it harder to compare high quality nests than to reject poor nests. 
- Sensing: foragers detect nesting sites at 1 unit of distance.

- Interaction: foragers do not interact, except in that they compete indirectly to collect resources from the initial colony.

- Stochasticity: nesting sites are randomly distributed throughout the environment, except in the close vicinity of the initial colony. The model allows controlling for a potential effect of this random distribution by comparing it with an equidistant distribution around the initial colony (see Table S1 and Results). During the exploration phase, foragers wander randomly in the environment.

- Observation: at each step of the exploration phase, the identity of foragers having visited each nest, the identity of nests visited by each forager and the nest chosen by each forager are recorded. At the end of the simulation, the number and size of newly produced colonies are recorded, as well as the qualities of all nests and of the chosen nests, and the distances from the initial colony to all nest and to chosen nests.

\section{Initialization}

The initial colony is located at the centre of a grid (61 x 61 patches). Exploration and allocation phases start with all foragers at the initial colony.

\section{$\underline{\text { Input data }}$}

The model does not use input data, but it is later tested against empirical data (see Model analysis below)

\section{Submodels}

Foragers follow one of three choice strategies: The first strategy, current_vs_previous_best, is the default strategy. It is used in all simulations unless specified otherwise.

-Current_vs_previous_best: when a forager visits a nesting site, she compares its estimated quality with the memorised quality of the nest she has chosen so far, and retains in memory only the quality and location of the best of the two. That is, each forager chooses a nesting site through serial comparisons between two nesting sites only, the one she is currently visiting and the best she has visited until then. This very simple strategy merely assumes that a forager can compare a nesting site with a memorised one, and select the best of the two. This seems reasonable given that ants have a good memory. For instance, they can memorise an odour associated with a food reward after a single exposure, and this memory can last up to three days even with no reinforcement (Piqueret et al. 2019).

- Best_of_last_ $n$ is an alternative strategy. Here, each forager memorises the last $n$ nesting sites she has visited (i.e. she has small or large memory when $n$ is small or large, respectively), and she chooses the best of these at the end of the exploration phase. This strategy is cognitively more demanding than current_vs_previous_best as foragers need to memorise many nesting sites, and its performance strongly depends on memory. The two strategies differ only in that current_vs_previous_best makes the choice progressively, on a running basis, whereas best_of_last_ $n$ makes it at the end of the exploration phase. Although it is cognitively more demanding, the latter strategy should match the former when $n$ is high but should otherwise fare worse.

- Random_choice is a control strategy. Each forager memorises all the nesting sites she visits, and she randomly chooses one at the end of the exploration phase. 


\section{Model analysis: parameter inference}

We use a parameter inference procedure to determine the values of the parameters under the condition that the model output approximates the observed data. In particular, we are interested in determining the relative credibility of the parameter values. Specifically, we use the Approximate Bayesian Computation (ABC) algorithm APMC (Lenormand et al. 2013). It estimates the posterior probability density over the parameters given the data, hereafter referred to as the parameter posterior density. It associates a positive value, the density, to every possible value of the parameter. $f_{X_{1}, X_{2}, \ldots \mid Y=Y_{0}}\left(x_{1}, x_{2}, \ldots\right)$ is the density for parameters $X_{1}=x_{1}, X_{2}=x_{2}, \ldots$ where $Y$ is the model output and $y_{0}$ the observed data. Higher densities mean higher credibility that the corresponding parameter values generate simulation output $\mathrm{Y}$ close to observed data $\mathrm{y}_{0}$.

The values of four parameters of interest are determined through the $A B C$ method (Table 1), whereas that of other parameters are held fixed at realistic a priori values (Table S1). The parameters of interest are the number of potential nesting sites (number_nests) which is an environmental parameter, the percentage of foragers (percentage foragers) which is a colony level trait, and two individual level traits which are the error foragers make when estimating the quality of a nesting site (nest_quality_assessment_error) and the knowledge of the environment they gain (number of nesting sites visited) while foraging during the weeks preceding colony fission (exploring_phase)

We carried out 19 independent parameter inferences, based on the observed data for each of the 19 colonies whose fission was recorded in the field (Chéron et al. 2011). Each of these colony fission events is characterised by the size of the initial colony before fission, its size after fission (some initial colonies survived fission while others did not), the investment of the initial colony in new colonies (the entirety or a fraction of its individuals), and the size of the new colonies (Table S2). For each independent parameter inference we set size_initial_colony and amount_allocated equal to the size of the initial colony before fission and to its investment in new colonies (i.e. summed size of all new colonies), respectively, and we study the parameter posterior density for one parameter at a time, by plotting the density as a function of the parameter (Table S2). The calibration is carried out by minimising the Euclidian distance between the vectors of simulated and observed new colony sizes. Performing an independent parameter inference for each colony helps us determine if the different reallocation patterns are likely to stem from different colony traits reflected in the parameter values.

\section{Posterior predictive checks}

As sanity checks for the inference procedure, we performed posterior predictive checks. For each of the 19 calibrations performed above, we drew 1,000 parameter sets from the parameter posterior distribution, ran the model with each, and checked that the observed data lie in the distribution resulting from the 1,000 simulation outputs. This procedure is not a proper model validation method, but a mere verification that the inference results are sound. It is necessary as the posterior density itself does not tell us if the model set with parameter values drawn from the parameter posterior distribution actually reproduces the data. A standard validation procedure is cross validation. It consists in performing the parameter inference with part of the data only, and testing it against the remaining part. However, since we perform one inference for each colony we have one data point for each inference only, hence this procedure cannot be applied. We used OpenMole 10.0 (Reuillon et al. 2013) to run the $A B C$ method. We ran the simulations on the European Grid Infrastructure (http://www.egi.eu/) and on the MeSU platform at Sorbonne-Université. We used R 3.6.1 (R Core Team 2019; RStudio Team 2020) for data analysis. 


\section{RESULTS}

The posterior predictive checks show that our simple model reproduces reasonably well the empirical data with the default choice strategy "current_vs_previous_best": for each of the 19 colonies, the observed data lies within or close to the $1^{\text {st }}$ and $3^{\text {rd }}$ quartiles computed from the 1,000 model outputs (Fig. 2). This is despite the large differences between fissioning colonies, in size (from 252 to 1,808 workers), investment into new colonies (allocating from 29.4 to $100 \%$ of their workers), and allocation pattern (producing one to seven new colonies) (Table S2). The number of simulated new nests tends to slightly exceed the number of observed new nests, but the few simulated nests produced in excess receive little resources only (Fig. 2).

In addition, the $A B C$ analysis determines the effects and credible values of the four parameters of special interest (Fig. S1). It shows that, for most colonies, the most important factors for simulated patterns of allocation to approach empirical patterns were a relatively low percentage of foragers (lower than ca 20\%) and/or few nesting sites (lower than ca 10). In some colonies, the number of foragers may be of a dozen or so, which straightforwardly constrains their choice to a few nesting sites given that each forager allocates resources to one nesting site only. In addition, colonies 5 and 8 , which produced the highest number of new colonies (seven and six), are well simulated when they are limited in nesting sites and/or when foragers have a poor knowledge of their environment (exploring_phase shorter than ca 1,000 steps while foragers need ca 3,000 steps to discover half of the nesting sites, Fig. S2). In contrast, colony 3, which produced two new colonies, is well simulated when it is limited in foragers and/or when foragers have a good knowledge of the environment (long exploring_phase), so that they may independently choose the same nests.

Nest_quality_assessment_error is the least important factor, except for colony 9. Note that changing the range of possible values for one parameter may change the values of the others. For instance, if number_nests is set to vary between 20 and 80 (instead of between 2 and 80 , Table 1) percentage foragers becomes lower and the simulated pattern of allocation remains close to the empirical pattern (not shown).

We checked whether the spatial distribution of nesting sites may affect the results of our model by comparing the pattern of allocation simulated when nesting sites are distributed randomly vs. placed equidistant around the initial colony. We found no difference (Fig. S3). This may be because nest density ( 2 to 80 nesting sites over 3,721 patches) and the distance of detection of a nest ( 1 unit) are such that the probability of a forager's path to come close to a nesting site is sufficiently low for foragers to have time to disperse throughout the environment before they find one, so that their starting location has no effect.

In addition, we compared the pattern of resource allocation simulated when foragers follow the default current_vs_previous_best choice strategy with that produced when they follow the cognitively more demanding best_of_last_n strategy (see Submodels in Material and Methods). In that case, each forager memorises up to $n$ nesting sites during the exploration phase, and she chooses the best of these at the end of this phase. As expected, when $n=1$, each forager remembers only the last nest she has visited and this strategy produces a pattern similar to the control stategy of random_choice, with many nesting sites receiving little resources each. As $n$ increases, fewer nesting sites are chosen and they receive more resources each, that is, the pattern of allocation increasingly resembles that produced by current_vs_previous_best. Last, when $n=$ number_nests foragers have the capacity to remember all the nesting sites and the strategies best_of_last_ $n$ and 
current_vs_previous_best yield identical patterns (Fig. S4). That is, best_of_last_n matches current_vs_previous_best at best and never outperforms it. This shows that

current_vs_previous_best is an efficient strategy albeit simple and cognitively little demanding.

\section{DISCUSSION}

Our simple individual-based model produces a pattern of resource allocation (number and size of new colonies) that is broadly consistent with the pattern observed empirically (Chéron et al. 2011). That is, simulated foragers allocate resources to new colonies as real foragers do in nature. Simulated foragers do so without coordinating or communicating in any way with one another, but by acting solitarily and relying on private information only. This differs from the process of colony relocation by house-hunting ants, where a consensus decision is made through a quorum, and the recruitment of scouts by tandem runs, but is consistent with the recent finding that brood transport can also be organised based on private information only and with no coordination between workers (Mersch et al. 2018). It suggests that resources allocation during colony fission is a different problem than house-hunting, and that in this context decisions may be taken independently by each forager rather than through consensus building.

A possible explanation for the difference in decision making between colony fission and househunting is that in the former foragers do not need to agree on selecting one nest only. On the contrary, their very aim is to select several nesting sites and engineer the split of the initial colony. It is possible that individual ants are cognitively not capable of tracking and adjusting resources allocation between various new colonies, so that this process may be achieved by being divided into simpler separate allocation tasks, for which no coordination is required. Another possible explanation for the difference is that colony fission has been studied in the field whereas househunting has largely been studied in the laboratory. The complexity of the natural environment may require more time than in the laboratory to assess and find a suitable nesting sites, and it may be that multiple independent assessments and choices is the most efficient strategy under these conditions. In addition, speed of decision is presumably not important for colony fission in C. piliscapa because foragers have plenty of time to visit and assess the quality of potential nesting sites before the onset of colony fission.

The $A B C$ analysis suggests that the number of potential nesting sites is more likely to range in the few tens, but we cannot compare this with empirical data as we are unable to determine in the field what is a putative nesting site for our model species $C$. piliscapa. Similarly, it suggests that the percentage of foragers is more likely to be below ca $20 \%$, which seems realistic for most social insects.

The model hypothesises that foragers assess potential nesting sites when they encounter them by chance while foraging, and later use this knowledge when the time to fission has come. This anticipation is supported by laboratory experiments of colony relocation of the ant $T$. albipennis. They have shown that foragers gather knowledge of their environment when foraging: even though their colony is not about to relocate they assess the quality of potential nesting sites they encounter (Franks et al. 2007). In C. piliscapa, foragers may gather knowledge of the environment while foraging for food in the weeks prior to colony fissioning. The model does not precisely simulate this. It merely generates a more or less pronounced heterogeneity of knowledge across foragers, and the 
number of steps (ticks) in the model is not connected to the real world. The results only highlight that, as expected, a minimal amount of time is required for foragers to explore their environment and assess nesting sites (Fig. S2).

Our model could be modified in several ways. C. piliscapa colonies adjust the number and size of new colonies produced to the intensity of competition (Cronin et al. 2012). Although our model simulates a single mature colony reproducing, it may provide a simple mechanism for this. When colony density is high in a population, many mature colonies may seek suitable nesting sites. Once a colony has started transporting resources to a nesting site it becomes unavailable to other colonies, so that each colony may be limited to fewer nesting sites and hence produce fewer (and larger) new colonies. Conversely, when colony density is low each mature colony may find more nesting sites and thus produce more (smaller) colonies. The model could be modified to include several mature colonies fissioning and competing for nesting sites. A second possible modification would be to include a threshold of quality for the acceptance of a nesting site. There is some evidence that househunting foragers have an intrinsic threshold of nest suitability, below which they reject a nest (Robinson et al. 2014). This could easily be implemented, including a heterogeneity of threshold between foragers as in (Cronin 2018), with some more selective than others. A third possible modification would be to add a quorum of visiting foragers for a nest to be selected, as occurs in house-hunting Temnothorax ants (Visscher 2007). Last, the model could also include the resorption of some new colonies, as observed in the field (Chéron et al. 2011). It may be that these colonies had failed to reach a threshold size of viability, and resorption of colonies smaller than a threshold could be implemented. Modifying the model would likely increase its already reasonably good with empirical data. Indeed, adding a quorum or the resorbtion of small colonies would decrease the number of small colonies produced by the model, which is the main difference between simulated and empirical data (Fig. 2). A caveat, however, is that modifying the model would require adding variables that we are currently unable to quantify. Aquiring more empirical knowledge of colony fission should be a priority for future studies.

The capacity of our model to reproduce well the observed data supports the parsimonious hypothesis that simple mechanisms may underlie the process of colony fission. However, this does not demonstrate that the modelled mechanism of workers acting solitarily without coordination reflects the biological mechanisms, and implementing some level of communication between foragers may be justified. Indeed, it has been shown in ants (Wendt et al. 2019) and bumblebees (Incorvaia et al. 2021) that trophallaxis between returning and outbound foragers may inform outbound foragers of the quality of the food sources available outside, and that this lowers their acceptance of food sources of lower qualities and increase their search for higher-quality food. In the context of colony fission, foragers that have visited nesting sites may communicate some information regarding their quality, and this knowledge could affect other foragers' selectivity and ultimately choice. Implementing communication in the model and comparing the performances of models with and without communication would be an interesting way to explore further the importance of solitary decisions in the process of resource allocation during colony fission. Nevertheless, the good performance of our model suggests that resource allocation may rely in part on solitary decisions based on private information. Indeed, recent works have shown that ant foragers visiting potential nesting sites combine private and social information before choosing one nesting site (Cronin 2013; Stroeymeyt et al. 2017). Our model investigates the partitioning of resources among new colonies 
(number and size of new colonies produced) but not the onset of colony fission nor its termination (i.e. when part of the initial colony survives colony fission). Whether these may be determined by solitary decisions or may require coordination remains to be studied. Empirical studies of colony fission are necessary to go further and should focus on both individual and colony level behaviours.

\section{REFERENCES}

Boulay R, Aron S, Cerdá X, et al (2017) Social Life in Arid Environments: The Case Study of Cataglyphis Ants. Annu Rev Entomol 62:305-321. https://doi.org/10.1146/annurev-ento-031616-034941

Burns DDR, Franks DW, Parr C, et al (2020) A longitudinal study of nest occupancy, trail networks and foraging in a polydomous wood ant population. Insectes Soc 67:419-427. https://doi.org/10.1007/s00040-020-00777-2

Burns DDR, Franks DW, Parr C, Robinson EJH (2021) Ant colony nest networks adapt to resource disruption. J Anim Ecol 90:143-152. https://doi.org/10.1111/1365-2656.13198

Chéron B, Cronin AL, Doums C, et al (2011) Unequal resource allocation among colonies produced by fission in the ant Cataglyphis cursor. Ecology 92:1448-1458. https://doi.org/10.1890/10-2347.1

Collett M, Collett TS, Bisch S, Wehner R (1998) Local and global vectors in desert ant navigation. Nature 394:269-272. https://doi.org/10.1038/28378

Cronin AL (2013) Conditional use of social and private information guides house-hunting ants. PLoS One 8:e64668. https://doi.org/10.1371/journal.pone.0064668

Cronin AL (2018) An agent-based model of nest-site selection in a mass-recruiting ant. J Theor Biol 455:54-63. https://doi.org/10.1016/j.cpr.2018.01.005

Cronin AL, Fédérici P, Doums C, Monnin T (2012) The influence of intraspecific competition on resource allocation during dependent colony foundation in a social insect. Oecologia 168:361369. https://doi.org/10.1007/s00442-011-2098-6

Cronin AL, Molet M, Doums C, et al (2013) Recurrent evolution of dependent colony foundation across eusocial insects. Annu Rev Entomol 58:37-55. https://doi.org/10.1146/annurev-ento120811-153643

Czaczkes TJ, Czaczkes B, Iglhaut C, Heinze J (2015) Composite collective decision-making. Proc R Soc B 282:20142723. https://doi.org/10.1098/rspb.2014.2723

Czaczkes TJ, Salmane AK, Klampfleuthner FAM, Heinze J (2016) Private information alone can trigger trapping of ant colonies in local feeding optima. J Exp Biol 219:744-751. https://doi.org/10.1242/jeb.131847

Dechaume-Moncharmont F-X, Dornhaus A, Houston Al, et al (2005) The hidden cost of information in collective foraging. Proc R Soc London B Biol Sci 272:1689-1695. https://doi.org/10.1098/rspb.2005.3137

Dussutour A, Nicolis SC (2013) Flexibility in collective decision-making by ant colonies: tracking food across space and time. Chaos, Solitons and Fractals 50:32-38. https://doi.org/10.1016/j.chaos.2013.02.004

Franks NR, Hooper JW, Dornhaus A, et al (2007) Reconnaissance and latent learning in ants. Proc R Soc London B Biol Sci 274:1505-1509. https://doi.org/10.1098/rspb.2007.0138

Franks NR, Pratt SC, Mallon EB, et al (2002) Information flow, opinion polling and collective intelligence in house-hunting social insects. Philos Trans R Soc Lond B Biol Sci 357:1567-1583. https://doi.org/10.1098/rstb.2002.1066

Grimm V, Berger U, DeAngelis DL, et al (2010) The ODD protocol: A review and first update. Ecol Modell 221:2760-2768. https://doi.org/10.1016/j.ecolmodel.2010.08.019 
Grüter C, Czaczkes TJ, Ratnieks FLW (2011) Decision making in ant foragers (Lasius niger) facing conflicting private and social information. Behav Ecol Sociobiol 65:141-148.

https://doi.org/10.1007/s00265-010-1020-2

Incorvaia DC, Hintze A, Dyer FC (2021) Spatial allocation without spatial recruitment in bumblebees. Behav Ecol 32:265-276. https://doi.org/10.1093/beheco/araa125

Lenoir A, Quérard L, Pondicq N, Berton F (1988) Reproduction and dispersal in the ant Cataglyphis cursor (Hymenoptera, Formicidae). Psyche (Stuttg) 95:21-44. https://doi.org/10.1155/1988/54685

Lenormand $M$, Jabot F, Deffuant G (2013) Adaptive approximate Bayesian computation for complex models. Comput Stat 28:2777-2796. https://doi.org/10.1007/s00180-013-0428-3

Mersch DP, Eckmann JP, Crespi A, Keller L (2018) Synchronised brood transport by ants occurs without communication. bioRxiv 364273. https://doi.org/10.1101/364273

Piqueret B, Sandoz JC, D'Ettorre P (2019) Ants learn fast and do not forget: Associative olfactory learning, memory and extinction in Formica fusca. R Soc Open Sci 6:. https://doi.org/10.1098/rsos.190778

Pratt SC, Mallon EB, Sumpter DJT, Franks NR (2002) Quorum sensing, recruitment, and collective decision-making during colony emigration by the ant Leptothorax albipennis. Behav Ecol Sociobiol 52:117-127. https://doi.org/10.1007/s00265-002-0487-x

R Core Team (2019) R: A language and environment for statistical computing. The R Foundation for Statistical Computing

Reuillon R, Leclaire M, Rey-Coyrehourcq S (2013) OpenMOLE, a workflow engine specifically tailored for the distributed exploration of simulation models. Futur Gener Comput Syst 29:1981-1990. https://doi.org/10.1016/j.future.2013.05.003

Richardson TO, Mullon C, Marshall JAR, et al (2018) The influence of the few: A stable 'oligarchy' controls information flow in house-hunting ants. Proc R Soc B Biol Sci 285:20172726. https://doi.org/10.1098/rspb.2017.2726

Robinson EJH, Feinerman O, Franks NR (2014) How collective comparisons emerge without individual comparisons of the options. Proc R Soc B Biol Sci 281:. https://doi.org/10.1098/rspb.2014.0737

Robinson EJH, Franks NR, Ellis S, et al (2011) A simple threshold rule is sufficient to explain sophisticated collective decision-making. PLoS One 6:e19981. https://doi.org/10.1371/journal.pone.0019981

Robinson EJH, Smith FD, Sullivan KME, Franks NR (2009) Do ants make direct comparisons? Proc R Soc B Biol Sci 276:2635-2641. https://doi.org/10.1098/rspb.2009.0350

RStudio Team (2020) RStudio: Integrated Development Environment for R. RStudio, Inc, Boston, MA RStudio, PBC, Boston, MA. https://doi.org/URL http://www.rstudio.com/.

Sasaki T, Pratt SC (2018) The psychology of superorganisms: collective decision making by insect societies. Annu Rev Entomol 63:259-275. https://doi.org/10.1146/annurev-ento-020117043249

Stroeymeyt N, Giurfa M, Franks NR (2017) Information Certainty Determines Social and Private Information Use in Ants. Sci Rep 7:1-13. https://doi.org/10.1038/srep43607

Visscher PK (2007) Group decision making in nest-site selection among social insects. Annu Rev Entomol 52:255-275. https://doi.org/10.1146/annurev.ento.51.110104.151025

Wendt S, Strunk KS, Heinze J, et al (2019) Positive and negative incentive contrasts lead to relative value perception in ants. Elife 8:e45450. https://doi.org/10.7554/eLife.45450

Wilensky U, Evanston I (1999) NetLogo: Center for connected learning and computer-based modeling. Northwest Univ Evanston, Illinois, US 49-52 
Table 1 Studied model parameters with range of values

\begin{tabular}{|c|c|c|}
\hline PARAMETER & DESCRIPTION & $\begin{array}{l}\text { VALUES USED BY THE ABC } \\
\text { METHOD }\end{array}$ \\
\hline number_nests & $\begin{array}{l}\text { Number of potential nesting sites } \\
\text { (nests) present in the environment, i.e. } \\
\text { where a new colony can be founded }\end{array}$ & From 2 to 80 (uniform law) \\
\hline percentage foragers & $\begin{array}{l}\text { Percentage of size_initial_colony that } \\
\text { are foragers }\end{array}$ & From $3 \%$ to $100 \%$ (uniform law) \\
\hline $\begin{array}{l}\text { nest_quality_assess- } \\
\text { ment_error }\end{array}$ & $\begin{array}{l}\text { Error made by a forager when assessing } \\
\text { the quality of a nest }\end{array}$ & From $0 \%$ to $50 \%$ (uniform law) \\
\hline exploring_phase & $\begin{array}{l}\text { Duration of the phase of exploration, } \\
\text { during which foragers visit nests and as- } \\
\text { sess their quality }\end{array}$ & $\begin{array}{l}\text { From } 1,000 \text { to } 10,000 \text { steps } \\
\text { (ticks) (uniform law) }\end{array}$ \\
\hline
\end{tabular}




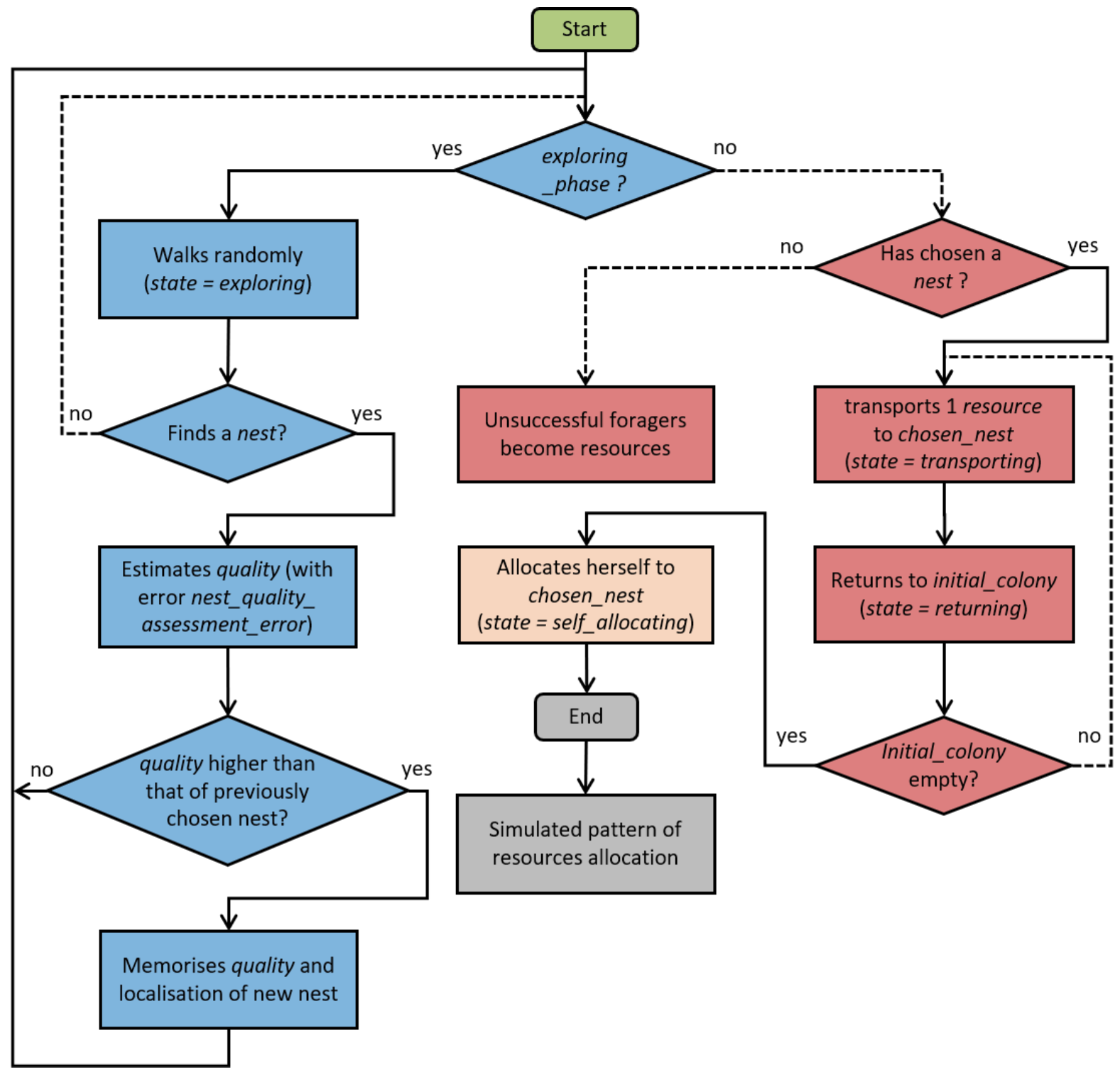

Fig. 1 Algorithm of the NetLogo model. The simulations start by an exploring_phase (blue) during which foragers randomly walk through the environment and visit nests they encounter. They memorise their quality, with an error of measure, and use this information to choose a nest. This exploring_phase mimics the knowledge of the environment that foragers obtain by foraging for food during the weeks that precede colony fission. Resources allocation to new colonies (red) starts after the exploring_phase. Foragers that failed to discover any nest become resources at the initial nest. Each forager that discovered a nest transports resources to the nest she has chosen. She transports one resource at a time, and repeatedly goes back and forth between her chosen nest and the initial_colony. When the initial_colony is depleted of resources, each forager allocates herself to her chosen nest (salmon pink), and the simulated pattern of resources allocation (grey) is recorded. 

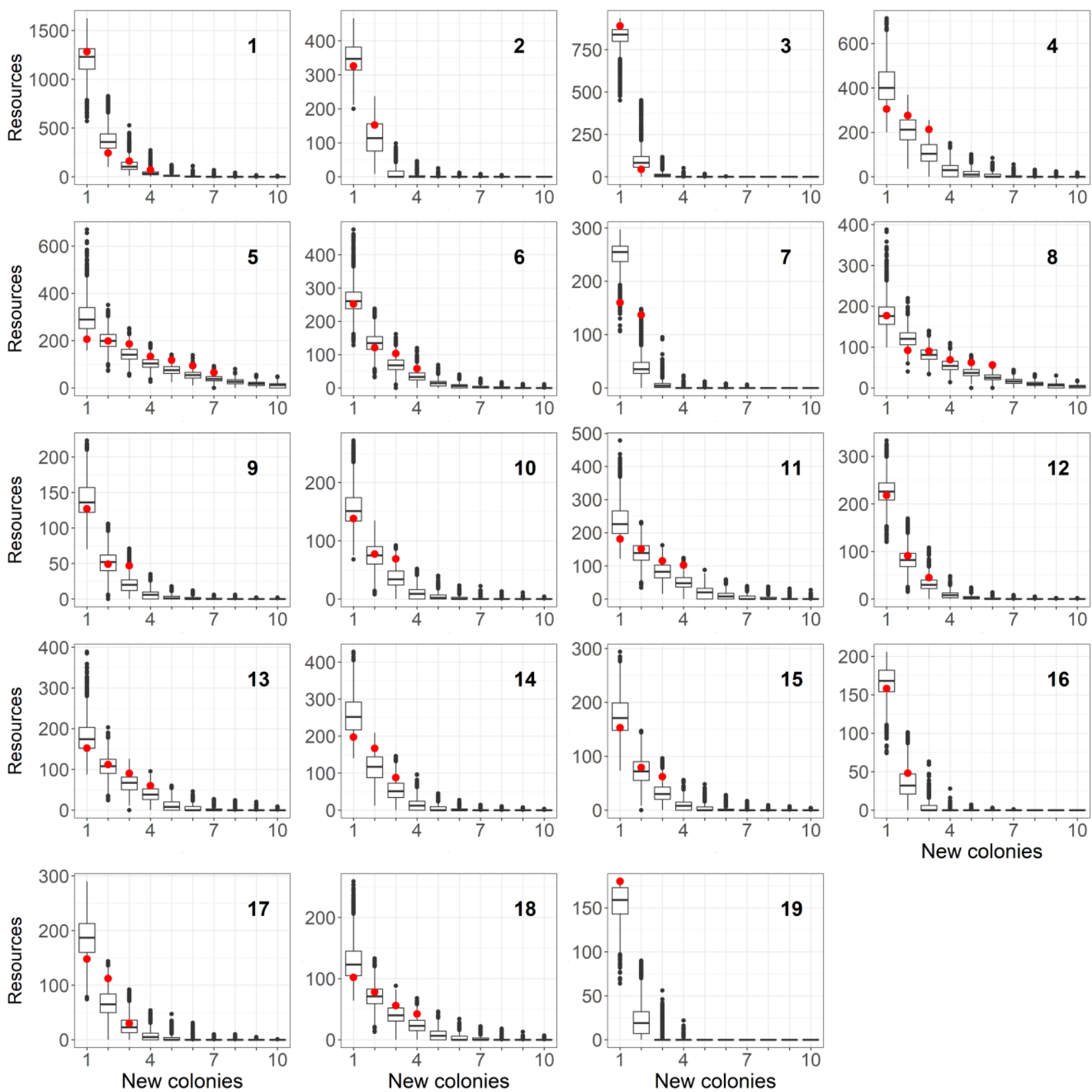

Fig. 2 Resource allocation after colony fission. Each panel shows the pattern of resource allocation (number and resources of new colonies) observed in nature (red dots) and simulated (black boxes), for each of the 19 colonies of varied size observed fissioning in nature (Table S1). Boxes represent the median and $1^{\text {st }}$ and $3^{\text {rd }}$ quartiles. The whiskers extend to the farthest data point within the limit of 1.5 times the distance between the $1^{\text {st }}$ and $3^{\text {rd }}$ quartiles, with outliers beyond this limit drawn as black dots. New colonies are ordered by decreasing size on the $x$-axis, from left to right. $n=1,000$ replicates per simulated colony. 
SUPPLEMENTARY MATERIAL FOR

Lavallée François, Chérel Guillaume, Monnin Thibaud (2021) No coordination required for resources allocation during colony fission in a social insect? An individual-based model reproduces empirical patterns. Animal Cognition

Table S1 Fixed model variables with range of values

\begin{tabular}{|c|c|}
\hline FIXED VARIABLES & DESCRIPTION AND VALUES \\
\hline patches & The environment is a grid of $61 * 61=3,721$ patches. \\
\hline initial_colony & The initial_colony is located at the centre of the grid. \\
\hline $\begin{array}{l}\text { nests_quality; } \\
\text { nests_quality_SD }\end{array}$ & $\begin{array}{l}\text { The quality of each nest is drawn from a normal distribution of mean nests_quality } \\
\text { (set to 70) and of standard deviation nests_quality_SD (set to 50). }\end{array}$ \\
\hline position_nests & $\begin{array}{l}\text { - Set to random, so that nests are positioned randomly in the environment, except } \\
\text { in a circle around the initial_colony that is empty of nesting sites (of radius } \\
\text { exclusion_radius set to } 10 \text { patches). } \\
\text { - The model also includes an equidistant positioning used for testing. Fig. S5 shows } \\
\text { that nests positioning has no effect }\end{array}$ \\
\hline
\end{tabular}

probability_of__ Set to unconditional, so that all foragers that have selected a nest transport resources transporting to it when colony fission starts, irrespective of the assessed quality of the chosen nest. The model includes 4 others functions not analysed here, where the probability of transporting may increase linearly, exponentially, logarithmically or logistically with the assessed quality of the chosen nest.

max_ticks Stops simulations if resource allocation is not completed after 20,000 ticks (never occurred). 
Table S2 Observed pattern of resource allocation (from Chéron et al 2011 Ecology) used for calibration. Each row gives the size (worker number) of one initial colony $(n=19)$ before colony fission, after colony fission, its total investment into new colonies (i.e. its size before minus its size after fission), and the number and size of new colonies produced by fission

\begin{tabular}{|c|c|c|c|c|c|c|c|c|c|c|}
\hline & $\begin{array}{l}\text { Initial } \\
\text { colony } \\
\text { before } \\
\text { fission }\end{array}$ & $\begin{array}{l}\text { Initial } \\
\text { colony } \\
\text { after } \\
\text { fission }\end{array}$ & $\begin{array}{l}\text { Investment of } \\
\text { the initial } \\
\text { colony in the } \\
\text { production of } \\
\text { new colonies }\end{array}$ & $\begin{array}{l}\text { New } \\
\text { colony } \\
1\end{array}$ & $\begin{array}{l}\text { New } \\
\text { colony } \\
2\end{array}$ & $\begin{array}{l}\text { New } \\
\text { colony } \\
3\end{array}$ & $\begin{array}{l}\text { New } \\
\text { colony } \\
4\end{array}$ & $\begin{array}{l}\text { New } \\
\text { colony } \\
5\end{array}$ & $\begin{array}{l}\text { New } \\
\text { colony } \\
6\end{array}$ & $\begin{array}{l}\text { New } \\
\text { colony } \\
7\end{array}$ \\
\hline Colony 1 & 1,808 & 49 & 1,759 & 1,284 & 244 & 160 & 71 & & & \\
\hline Colony 2 & 1,628 & 1,150 & 478 & 326 & 152 & & & & & \\
\hline Colony 3 & 1,328 & 394 & 934 & 890 & 44 & & & & & \\
\hline Colony 4 & 1,037 & 243 & 794 & 305 & 276 & 213 & & & & \\
\hline Colony 5 & 999 & 0 & 999 & 206 & 198 & 186 & 133 & 117 & 94 & 65 \\
\hline Colony 6 & 791 & 257 & 534 & 252 & 121 & 103 & 58 & & & \\
\hline Colony 7 & 789 & 492 & 297 & 160 & 137 & & & & & \\
\hline Colony 8 & 780 & 234 & 546 & 177 & 92 & 90 & 69 & 62 & 56 & \\
\hline Colony 9 & 720 & 497 & 223 & 127 & 49 & 47 & & & & \\
\hline Colony 10 & 577 & 293 & 284 & 138 & 77 & 69 & & & & \\
\hline Colony 11 & 549 & 0 & 549 & 181 & 151 & 115 & 102 & & & \\
\hline Colony 12 & 531 & 177 & 354 & 218 & 91 & 45 & & & & \\
\hline Colony 13 & 495 & 81 & 414 & 152 & 112 & 90 & 60 & & & \\
\hline Colony 14 & 452 & 0 & 452 & 197 & 167 & 88 & & & & \\
\hline Colony 15 & 294 & 0 & 294 & 153 & 79 & 62 & & & & \\
\hline Colony 16 & 291 & 85 & 206 & 158 & 48 & & & & & \\
\hline Colony 17 & 290 & 0 & 290 & 148 & 112 & 30 & & & & \\
\hline Colony 18 & 278 & 0 & 278 & 102 & 78 & 56 & 42 & & & \\
\hline Colony 19 & 252 & 72 & 180 & 180 & & & & & & \\
\hline
\end{tabular}




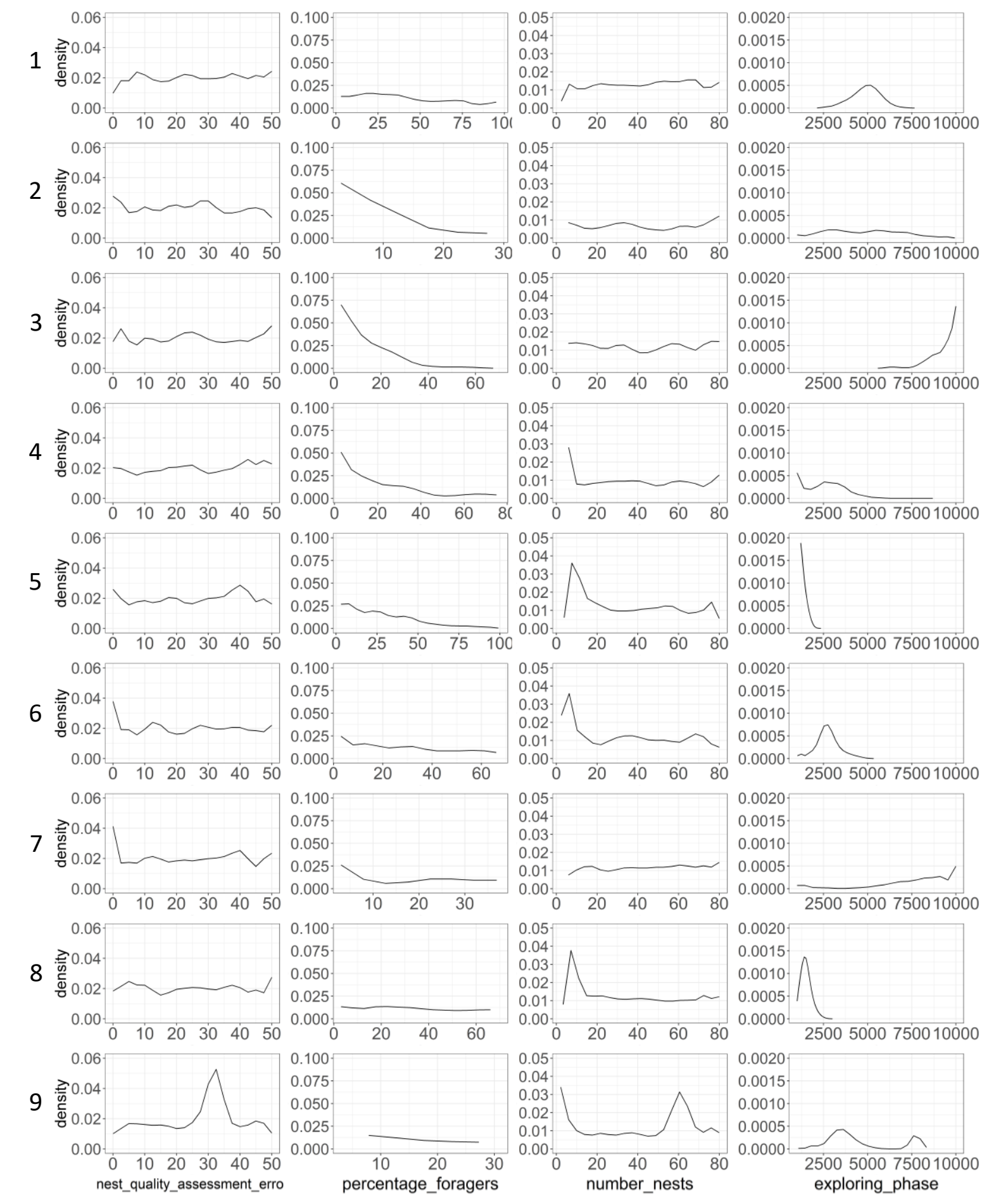

Fig. S1a Marginal values of the four parameters studied with the $A B C$ method for the first nine colonies. The density represents the likelihood that the values of the plotted parameter yield a pattern of simulated resources allocation similar to the empirical pattern. For instance, for colony 1 nest_quality_assessment_error, percentage_foragers and number_nests show no clearly more likely values whereas intermediate values of exploring_phase (around 5,000 steps) are more likely to yield a simulated pattern similar to the empirical one. Note that the range of values explored for percentage foragers vary across colonies (the number of foragers cannot exceed the amount of resources allocated hence percentage_foragers $\leq$ amount_allocated / size_initial_colony*100). 


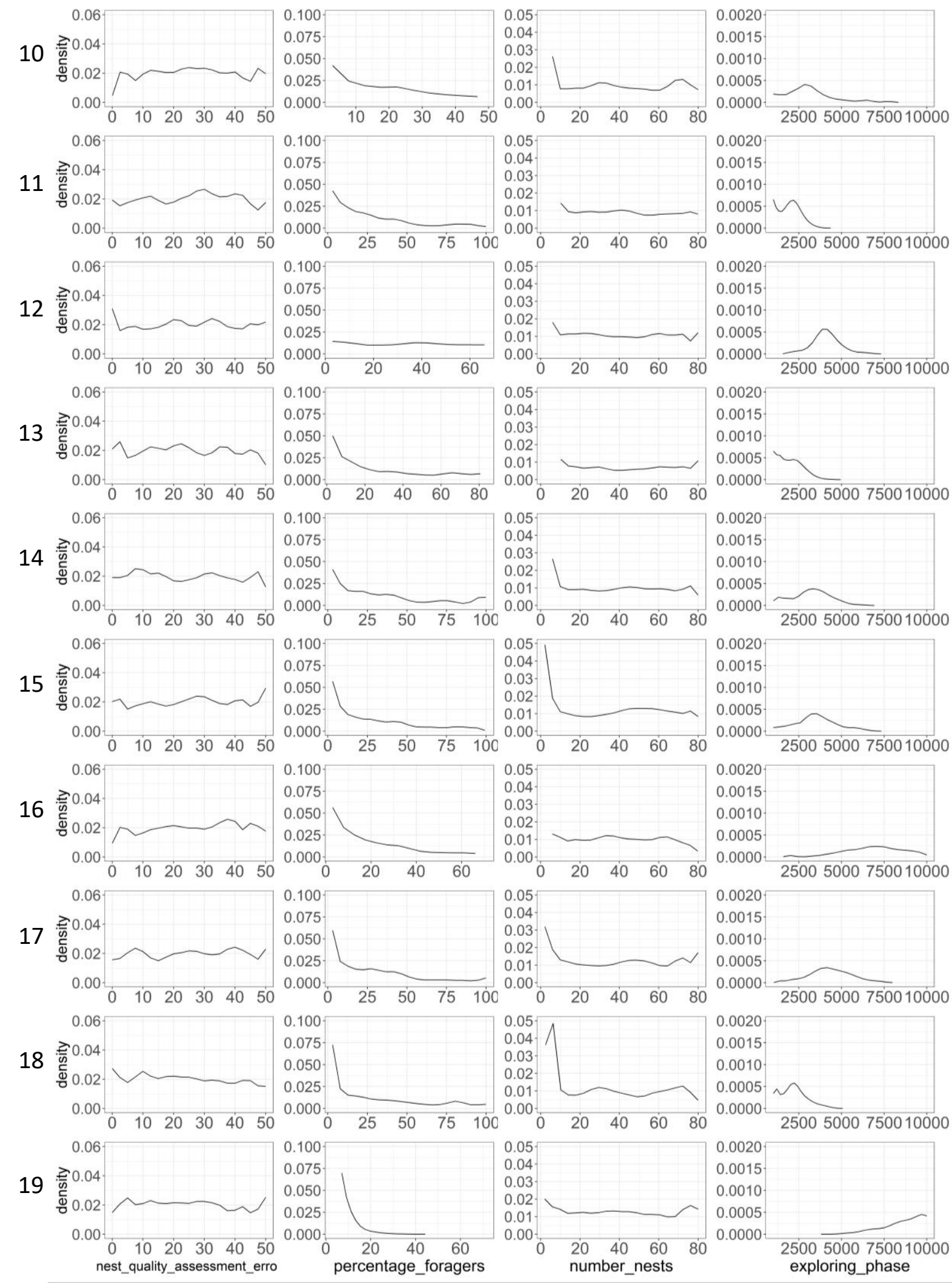

Fig. S1b Marginal values of the four parameters studied with the $A B C$ method for the last ten colonies (see legend previous page). 


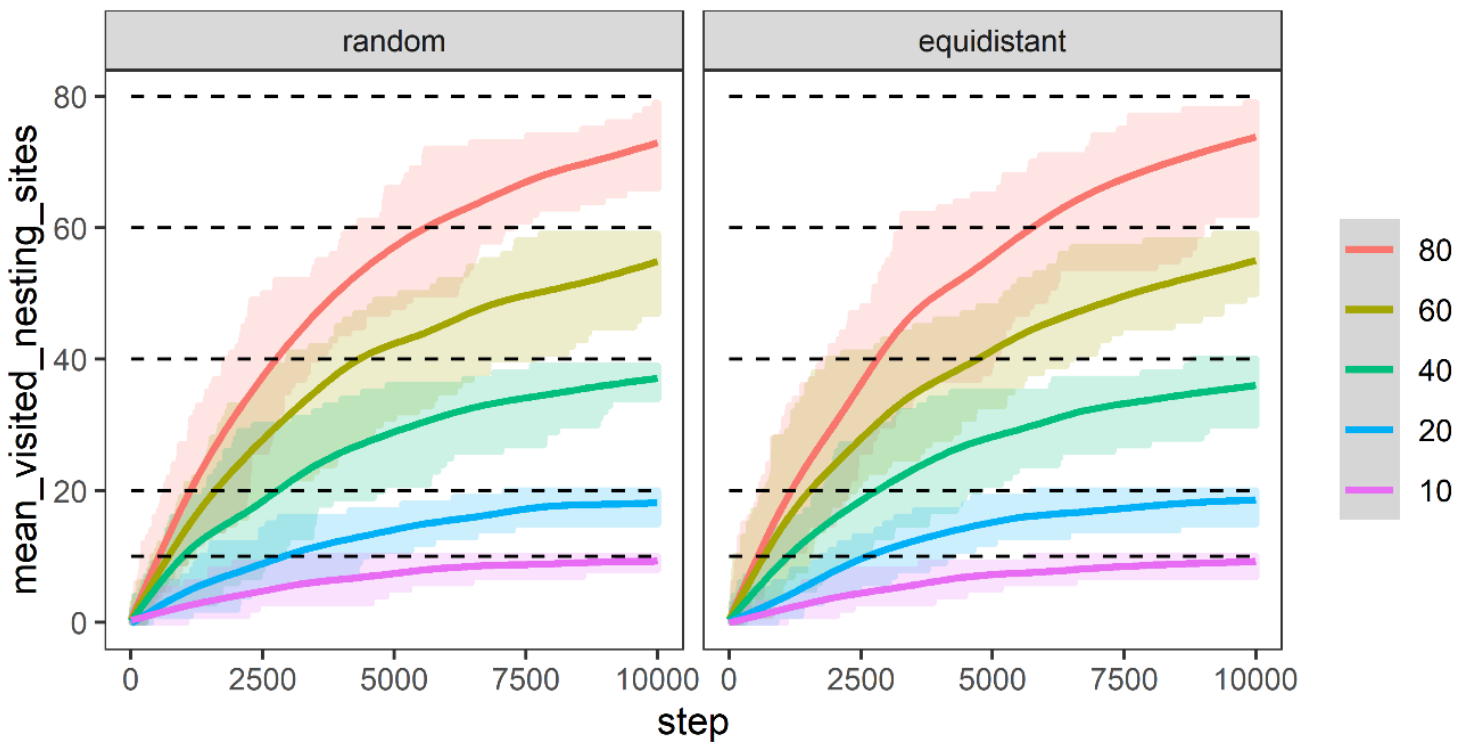

Fig. S2 Number of nesting sites visited by a forager over the duration of the exploring_phase. The environment contains 10 to 80 nesting sites (coloured lines), which are either distributed randomly (left panel) or equidistant around the initial nest (right, $n=20$ replicates per panel). The panels show (1) that a forager discovers nests at the same rate irrespective of their spatial distribution, and (2) that she has visited around half of the nesting sites at ca 3,000 steps. 

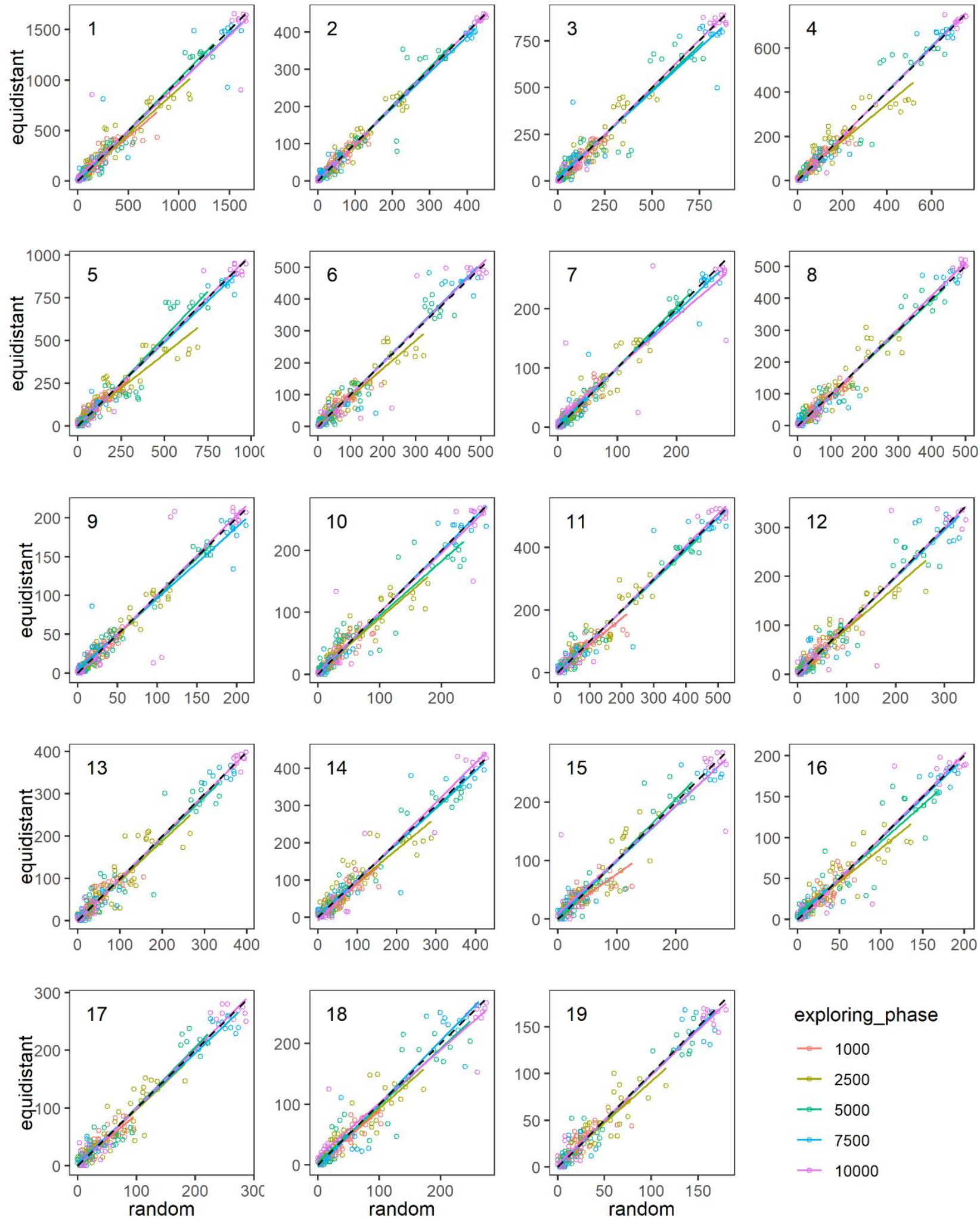

exploring_phase

$-1000$

$-2500$

$\rightarrow 5000$

$-7500$

$\rightarrow 10000$

Fig. S3 Effect of nest distribution on resource allocation for the 19 colonies simulated. Scatterplots show the size of new colonies produced when nesting sites distribution is random ( $x$ axis) vs. equidistant ( $y$ axis), for exploring_phase of varied lengths (1,000 to 10,000 steps). Dots are distributed along the bissector, and linear regression lines closely match the bissector, showing that nests distribution has no effects on the patter of resource allocation among new colonies. Simulations carried out with $20 \%$ of foragers, 50 nesting sites and no error in the estimation of nesting sites qualities. $n=10$ replicates per colony and per condition ( 5 durations of the exploring phase $* 2$ distribution of nests). 


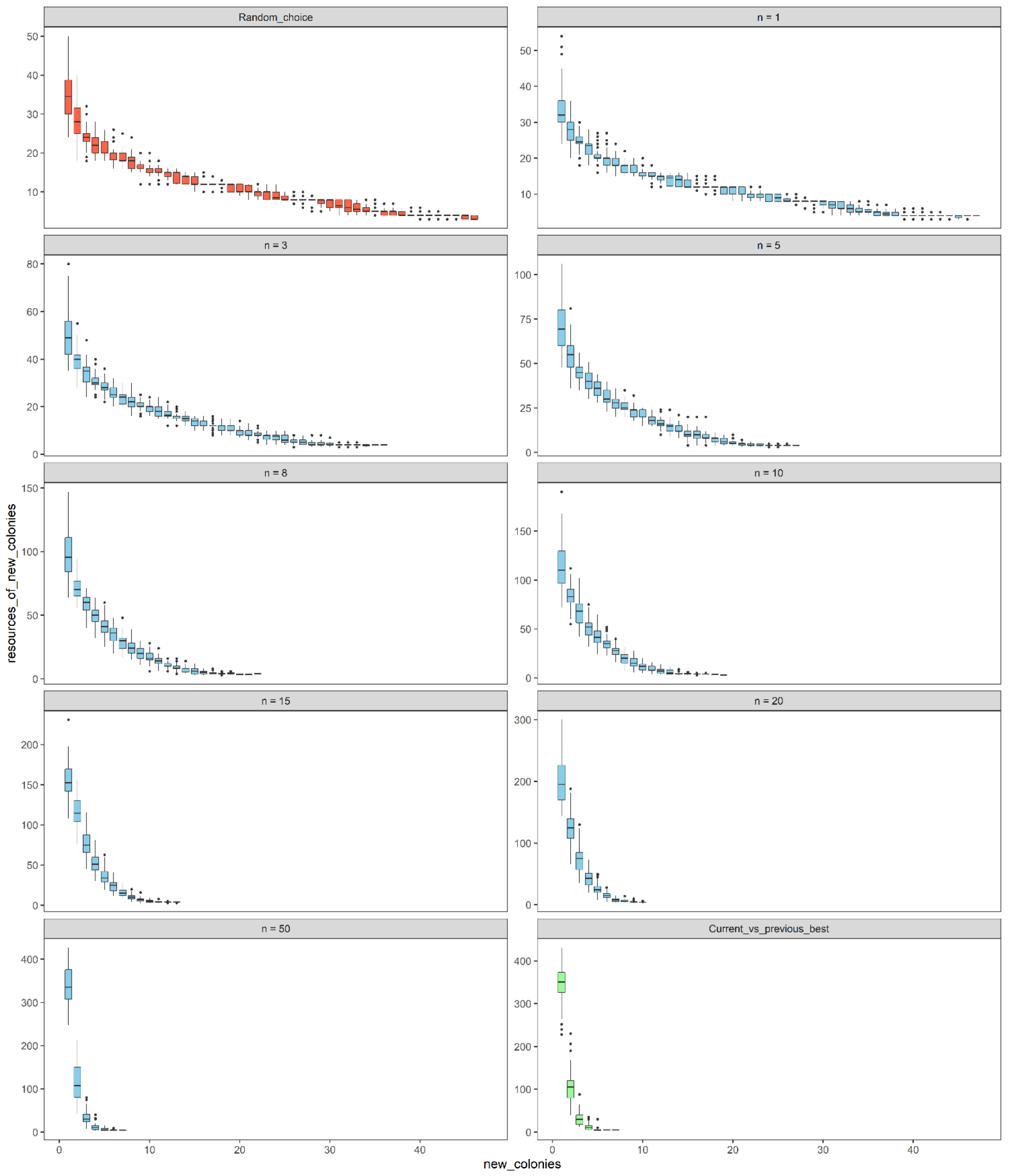

Fig. S4 Effect of choice strategies and of memory on resource allocation. Plots show the size of new colonies when foragers follow each of the three choice strategies, with new colonies ordered by decreasing size on the $x$-axis, from left to right. When foragers chose randomly they produce many new colonies of relatively comparable size (red, see y-axis) while when they follow the current_vs_previous_best strategy they produce few new colonies only with marked size differences (green). When foragers follow the best_of_last_ $n$ choice strategy (blue) the outcome is similar to random_choice when $n$ is low (they only remember the last or last few visited nesting sites), similar to current_vs_previous_best when $n$ is high (they remember most nesting sites) and internmediate when $n$ is intermediate. This shows the high effect of memory on best_of_last_ $n$ strategy, and the high performance of the cognitively less demanding current_vs_previous_best strategy. Simulations carried out with colonies of size 500, 20\% of foragers, 50 randomly distributed nests, no error in assessment of their quality and an exploring_phase of 5,000 ticks. $n=50$ replicates per panel. 